CARDIOVASCULAR MEDICINE

\title{
Myocardial performance index for assessment of left ventricular outcome in successfully recanalised anterior myocardial infarction
}

\author{
M Kato, K Dote, S Sasaki, K Goto, H Takemoto, S Habara, D Hasegawa
}

Heart 2005;91:583-588. doi: 10.1136/hrt.2004.035758

See end of article for authors' affiliations

Correspondence to:

Dr Masaya Kato,

Department of Cardiology,

Hiroshima City Asa

Hospital, 2-1-1

Kabeminami, Asakita-ku,

Hiroshima 731-0293

Japan; ms-katou@asa-

hosp.city.hiroshima.jp

Accepted 13 July 2004
Objective: To investigate whether the myocardial performance index (MPI) can predict left ventricular functional outcome in patients with early recanalisation after anterior acute myocardial infarction (MI) and to determine when the index should be measured.

Design: MPI was measured serially by two dimensional Doppler echocardiography after successful percutaneous coronary intervention (PCI). Left ventricular function was evaluated by echocardiography and left ventriculography. To assess coronary microvascular damage, the coronary flow velocity pattern was measured immediately after $\mathrm{PCl}$ with a Doppler guidewire.

Setting: Hiroshima City Asa Hospital.

Patients: 32 consecutive patients with their first anterior acute MI who had complete occlusion of left anterior descending coronary artery.

Interventions: Successful PCl within six hours of symptom onset.

Main outcome measures: Left ventricular anterior wall motion score index (A-WMSI), left ventricular end diastolic pressure (LVEDP), left ventricular ejection fraction (LVEF), and left ventricular end diastolic volume (LVEDV).

Results: There was a significant negative correlation between MPI on day 2 and the coronary diastolic deceleration time $(r=-0.66, \mathrm{p}<0.002)$, as well as a significant positive correlation with the coronary diastolic deceleration rate $(r=0.74, p<0.0001)$. MPI on day 2 was significantly correlated with the short and long term changes of A-WMSI and with the short term changes of LVEDP. Furthermore, MPI on day 2 was significantly correlated with the short and long term changes of LVEF $(r=-0.52, p<0.003$, and $r=-0.64, p<0.0008$, respectively) and of $\operatorname{LVEDV}(r=0.51, p<0.003$, and $r=0.41$, $\mathrm{p}<0.05$, respectively).

Conclusions: Doppler derived MPI on day 2, representative of the early coronary microvascular state, can predict the left ventricular functional outcome after early successful recanalisation of a patient's first anterior acute $\mathrm{Ml}$.
M yocardial salvage after reperfusion may be limited by deleterious changes in the microcirculation of the previously ischaemic tissue, which can result in a progressive decrease of capillary blood flow to potentially viable myocytes (the no reflow phenomenon). The acute loss of part of the myocardium also results in an abrupt increase of the load on the rest of the heart that induces myocardial remodelling. ${ }^{1}$ Studies ${ }^{2-5}$ have shown that the optimum time for estimating microvascular integrity by myocardial contrast echocardiography to predict myocardial viability may be one day after recanalisation in acute myocardial infarction (MI). These studies have shown that microvascular injury is already partially healed and neovascularisation is occurring within the infarcted area on day 2 after the event.

The myocardial performance index (MPI) is a simply measurable Doppler derived index of combined systolic and diastolic myocardial performance, which is reported to be useful for evaluating the prognosis after acute MI. ${ }^{6-9}$ The present study investigated whether the MPI can be used to predict left ventricular functional outcome in patients with early recanalisation after acute anterior MI, as well as the optimum time to measure the index for predicting left ventricular outcome.

\section{METHODS}

\section{Patient population and study design}

Among 70 consecutive patients with their first anterior acute MI and emergency percutaneous coronary intervention
(PCI), we enrolled 32 consecutive patients (24 men and eight women) aged from 45-82 years (mean (SD) age 66 (11) years) with a completely occluded proximal or middle segment of the left anterior descending coronary artery on emergency coronary angiography and who underwent successful PCI within six hours after the onset of symptoms. Acute MI was diagnosed on the basis of chest pain lasting for over 30 minutes, ST elevation of at least $2 \mathrm{~mm}$ in two adjacent leads on the ECG, and an increase of serum creatine kinase to more than twice the upper limit of normal. We excluded 13 patients with spontaneous coronary recanalisation, one patient with occlusion of the distal segment of the left anterior descending coronary artery, six patients who underwent PCI more than six hours after symptom onset, eight patients whose cardiac function could not be evaluated by emergency left ventriculography because of their poor haemodynamic function, and 10 patients with restenosis or reocclusion of the culprit lesion within six months after revascularisation. None of the enrolled patients had atrial fibrillation, atrial flutter, atrioventricular block, severe mitral or aortic valvar disease, or severe pericardial effusion. In all patients, MPI was measured serially just after, one day after,

Abbreviations: A-WMSI, anterior wall motion score index; LVEDP, left ventricular end diastolic pressure; LVEDV, left ventricular end diastolic volume; LVEF, left ventricular ejection fraction; $\mathrm{MI}$, myocardial infarction; $\mathrm{MPI}$, myocardial performance index; $\mathrm{PCl}$, percutaneous coronary intervention; TIMI, thrombolysis in myocardial infarction 
and one week after successful revascularisation. Left ventricular ejection fraction (LVEF) and end diastolic volume (LVEDV) were evaluated by left ventriculography just before, one week after, and six months after revascularisation. The coronary blood flow velocity pattern and coronary flow velocity reserve in the left anterior descending artery were measured to evaluate the coronary microvascular function in 21 patients immediately after PCI with a Doppler guidewire. These factors were measured only in patients whose PCI was finished within 120 minutes to avoid the excessive radiation exposure and loading of contrast media. We obtained written informed consent from each patient and our institutional ethics committee approved the study.

\section{Echocardiography}

Two examiners (MK and SS) performed complete two dimensional and Doppler echocardiographic examinations with a Sonos 5500 (Agilent Technologies, Andover, Massachusetts, USA). A parasternal long axis or short axis view at the mid left ventricular level was used to measure the left ventricular end systolic and end diastolic dimensions. The left ventricular wall was divided into 16 segments and the regional wall motion of each segment was examined and scored according to a modified recommendation of the American Society of Echocardiography, ${ }^{10}$ in which a score of 1 indicates a normal, 2 a hypokinetic, 3 an akinetic, and 4 a dyskinetic segment. Nine of the 16 segments were determined to be in the vicinity of the left anterior descending coronary artery and the anterior wall motion score index (A-WMSI) was calculated as an average of the wall motion scores of these nine segments. ${ }^{11}$ The change in A-WMSI was also estimated. The mitral inflow velocity pattern was recorded in the apical long axis view with the pulsed wave Doppler sample volume positioned at the tips of the mitral leaflets during diastole. The left ventricular outflow velocity pattern was also recorded in the apical long axis view with the pulsed wave Doppler sample volume positioned just below the aortic valve.

\section{Doppler measurements}

Five consecutive beats were measured and averaged for assessment of each parameter. Doppler timed intervals were measured from the mitral inflow and left ventricular outflow velocity time intervals (fig 1). The interval between mitral valve closure and opening corresponds to the time from cessation to onset of mitral inflow and is equal to the sum of the isovolumic relaxation time, the ejection time, and the isovolumic contraction time. Left ventricular ejection time was measured as the duration of the left ventricular outflow velocity profile. Then the MPI was calculated as the sum of isovolumic contraction time and isovolumic relaxation time divided by ejection time. These measurements were assessed blinded for clinical data and left ventricular function of each patient. The normal mean (SD) of MPI in 100 patients (45 men, mean (SD) age 65 (14) years) with no history of heart diseases, normal blood pressure, and normal two dimensional and Doppler echocardiography in our institute was $0.34(0.05)$.

\section{Acute catheterisation and angiography}

After administration of intravenous heparin (2000 U), coronary angiography and left ventriculography were performed by standard techniques. At first, baseline haemodynamic variables, including the left ventricular end diastolic pressure (LVEDP), were measured through the catheter. Then angiography was performed in multiple projections to visualise the infarct related artery. Left ventriculography was done before and at one week (8.8 (2.2) days) after PCI in all patients and at six months (5.8 (1.4) months) after PCI in 24

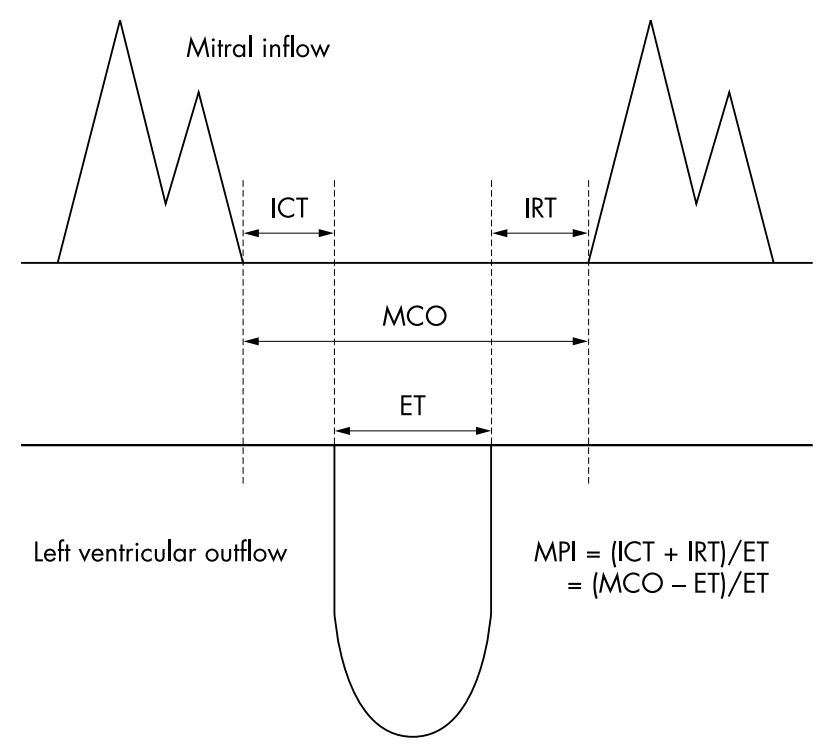

Figure 1 Schema for measurement of Doppler intervals. Myocardial performance index (MPI) is defined as the sum of isovolumic contraction time (ICT) and isovolumic relaxation time (IRT) divided by ejection time (ET). The sum of ICT and IRT is equal to the difference between the interval from cessation to onset of the mitral inflow (MCO) and ET.

patients. LVEF and LVEDV were calculated by the arealength method ${ }^{12}$ with correction for the $30^{\circ}$ right anterior oblique projection. To determine the method's reproducibility, two investigators measured the LVEF and LVEDV in 20 patients. The correlation was excellent between the first and the second day measurements (intraobserver variability) for each parameter $(\mathrm{y}=0.91 \mathrm{x}+4.05, r=0.99, \mathrm{p}<0.0001$ for LVEF; $y=0.92 \mathrm{x}+10.60, r=0.98, \mathrm{p}<0.0001$ for LVEDV). Analysis of interobserver variability in the measurement of each parameter also showed high reproducibility $(r=0.99$, $\mathrm{p}<0.0001$, respectively).

\section{Angioplasty}

PCI was performed according to the standard technique. All of the patients received another $5000 \mathrm{U}$ of heparin before angioplasty and additional heparin (2000 U) was administered at hourly intervals during the procedure. In all patients, angiography was successful. This was defined as TIMI (thrombolysis in myocardial infarction) grade 2 or 3 flow and $<50 \%$ diameter narrowing after PCI as shown by computer assisted quantitative coronary angiography performed with an online analysis system ${ }^{13}$ and digital imaging (ACA-DCI; Phillips, Andover, Massachusetts, USA).

\section{Analysis of coronary blood flow}

PCI (including stent placement) was successful in all patients. After recanalisation was completed, the guidewire was exchanged for a 0.014 inch intracoronary Doppler tipped flow wire (12 MHz, FloWire, Cardiometrics Inc, Mountain View, California, USA) to measure intracoronary flow in 21 patients. The tip of the wire was placed just distal to the site of recanalisation to ensure that the sampling volume corresponded to the site of occlusion. This allowed assessment of the blood flow to the entire region at risk. Doppler flow velocity spectra were analysed off line to determine the following parameters: peak and mean antegrade coronary flow velocity during systole and diastole, average peak velocity, and deceleration time of diastolic velocity (fig 2). The rate of decrease of the flow velocity during diastole was also calculated as the diastolic deceleration rate. Pathological rapid early systolic retrograde flow was defined as a negative 


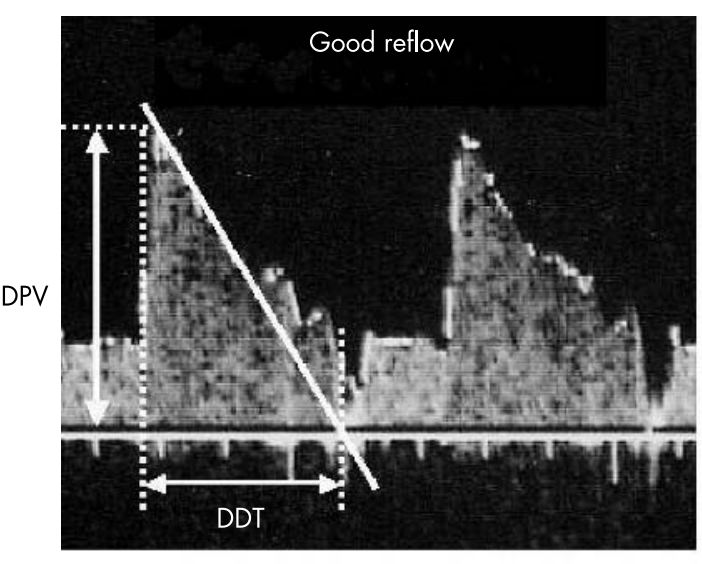

$\mathrm{DDR}=\mathrm{DPV} / \mathrm{DDT}$

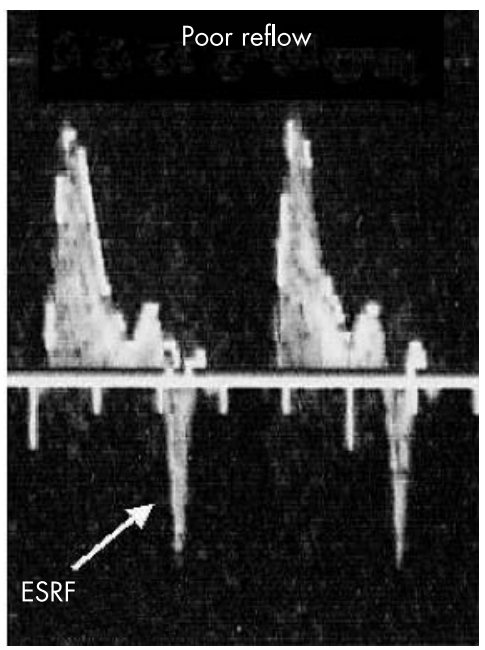

Figure 2 Schema for measurement of Doppler coronary flow velocity spectra. Peak antegrade coronary flow velocity during diastole (DPV) and deceleration time of diastolic velocity (DDT) are determined. The rate of decrease of flow velocity during diastole is calculated as the diastolic deceleration rate (DDR). Pathological rapid early coronary systolic retrograde flow (ESRF) is defined as a negative peak velocity $\geqslant 10 \mathrm{~cm} / \mathrm{s}$ and duration $\geqslant 60 \mathrm{~ms}$ (arrow).

peak velocity $\geqslant 10 \mathrm{~cm} / \mathrm{s}$ and duration $\geqslant 60 \mathrm{~ms} \cdot{ }^{14}$ All measurements were repeated three times and the mean values were calculated.

\section{Statistical analysis}

Data are expressed as the mean (SD). The $\chi^{2}$ test and the $t$ tests were used to compare categorical and continuous variables, respectively, between groups. Linear regression analysis was used to determine the relation between continuous variables. A probability value of $\mathrm{p}<0.05$ was considered to indicate significance.

\section{RESULTS}

\section{Patient characteristics}

Table 1 summarises baseline characteristics of 32 patients. Stents were placed in 29 patients. Two patients were treated with directional coronary atherectomy alone and one patient was treated with balloon angioplasty alone. No patients died during the first year after the onset of MI.

Table 1 Baseline characteristics of 32 patients

\begin{tabular}{ll}
\hline Age (years) & $66(11)$ \\
Men & $24(75 \%)$ \\
Elapsed time from symptom onset to reperfusion & $3.4(1.5)$ \\
(hours) & \\
Culprit site & $14(44 \%)$ \\
$\quad$ Proximal LAD & $18(56 \%)$ \\
Middle LAD & $3(9 \%)$ \\
Killip class $\geqslant \| 1$ & $3(9 \%)$ \\
IABP deployment & $20(63 \%)$ \\
Hypertension & $11(34 \%)$ \\
Diabetes mellitus & $18(56 \%)$ \\
Hyperlipidaemia & $12(38 \%)$ \\
Current smoking & \\
Oral drugs after PCI & $24(75 \%)$ \\
ACE inhibitor & $3(9 \%)$ \\
ARB & $1(3 \%)$ \\
$\beta$ Blocker & $1(3 \%)$ \\
Calcium antagonist & $1(3 \%)$ \\
Nitrate & $15(47 \%)$ \\
Statin & $31(97 \%)$ \\
Ticlopidine & $19(59 \%)$ \\
Aspirin &
\end{tabular}

Data are numbers of patients or mean (SD)

$\mathrm{ACE}$, angiotensin converting enzyme; $\mathrm{ARB}$, angiotensin II receptor blocker; IABP, intra-aortic balloon pump counterpulsation; LAD, left anterior descending artery; $\mathrm{PCl}$, percutaneous coronary intervention.
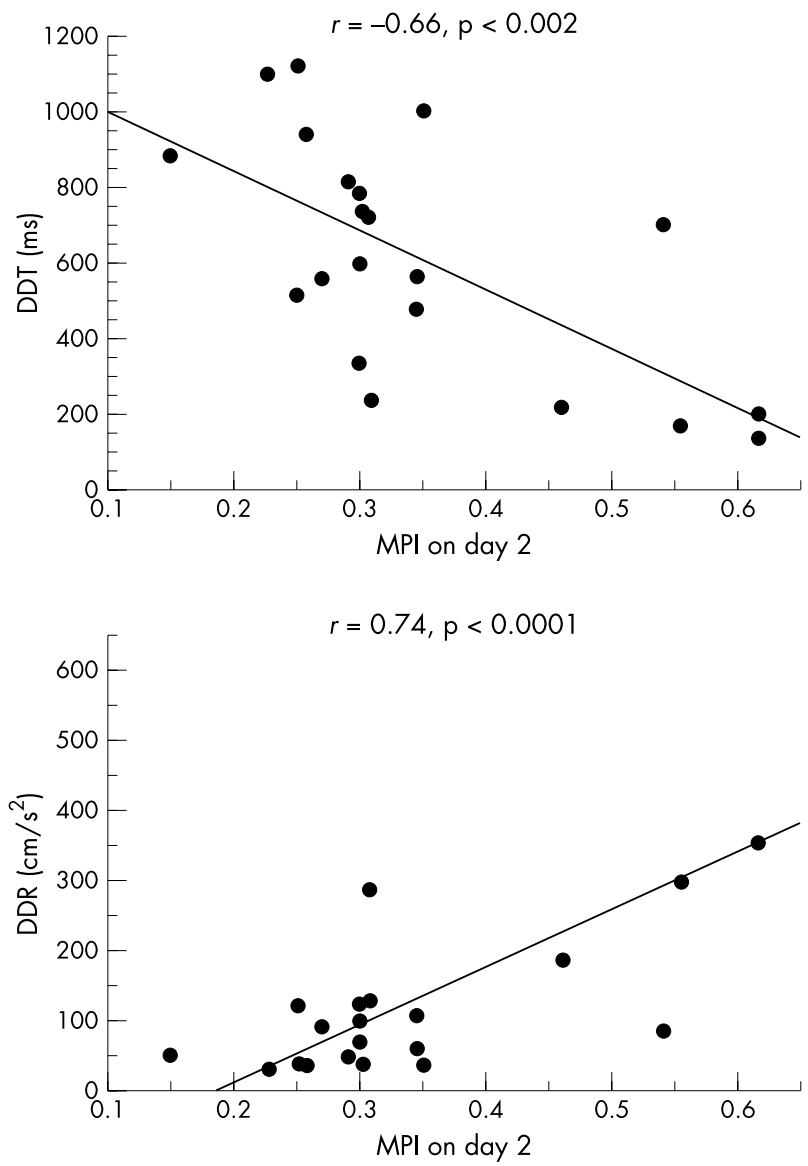

Figure 3 Correlation between MPI on day 2 and the coronary diastolic flow pattern. MPI on day 2 has a significant negative correlation with coronary diastolic deceleration time (DDT) but a significant positive correlation with coronary diastolic deceleration rate (DDR).

\section{MPI and coronary flow velocity patterns}

Mean (SD) MPI one day after PCI $(0.34$ (0.13)) was significantly less than at one week after PCI $(0.42(0.17))$. MPI just after PCI $(0.37(0.13))$ was similar to MPI on day 2 and day 7. Early systolic retrograde coronary flow was observed in nine of the 22 patients. In patients with early systolic retrograde coronary flow, MPI on day 2 after PCI was significantly larger than in patients without such flow $(0.43$ 
Table 2 Correlation between serial measurements of myocardial performance index (MPI) and left ventricular functional variables

\begin{tabular}{|c|c|c|c|c|c|c|}
\hline & \multicolumn{6}{|l|}{ MPI } \\
\hline & \multicolumn{2}{|c|}{ Just after $\mathrm{PCl}$} & \multicolumn{2}{|l|}{ Day 2} & \multicolumn{2}{|l|}{ Day 7} \\
\hline & $r$ & $\mathrm{p}$ Value & $r$ & $\mathrm{p}$ Value & $r$ & p Value \\
\hline Peak CK & 0.11 & 0.56 & 0.74 & $<0.0001$ & 0.29 & 0.11 \\
\hline Peak CK-MB & 0.004 & 0.98 & 0.71 & $<0.0001$ & 0.16 & 0.39 \\
\hline \multicolumn{7}{|c|}{ Percentage change in $\mathrm{A}-\mathrm{WMSI}$ after $\mathrm{PCl}$} \\
\hline After 1 week & 0.18 & 0.33 & 0.44 & $<0.02$ & 0.23 & 0.20 \\
\hline After 6 months & 0.11 & 0.60 & 0.62 & $<0.002$ & 0.30 & 0.14 \\
\hline \multicolumn{7}{|c|}{ Percentage change in LVEDP after PCI } \\
\hline After 1 week & 0.26 & 0.16 & 0.38 & $<0.04$ & 0.07 & 0.71 \\
\hline After 6 months & 0.18 & 0.41 & 0.16 & 0.45 & 0.14 & 0.52 \\
\hline \multicolumn{7}{|c|}{ Percentage change in LVEF after PCI } \\
\hline After 1 week & -0.24 & 0.19 & -0.52 & $<0.003$ & -0.32 & 0.07 \\
\hline After 6 months & -0.22 & 0.31 & -0.64 & $<0.0008$ & -0.29 & 0.16 \\
\hline \multicolumn{7}{|c|}{ Percentage change in LVEDV after PCI } \\
\hline After 1 week & 0.17 & 0.37 & 0.51 & $<0.003$ & 0.24 & 0.18 \\
\hline After 6 months & 0.03 & 0.87 & 0.41 & $<0.05$ & 0.16 & 0.47 \\
\hline
\end{tabular}

(0.13) v 0.29 (0.09), p < 0.007), whereas MPI immediately after PCI was similar in both groups $(0.41 \quad(0.13) \vee 0.36$ $(0.14), p=0.41)$. There was a significant negative correlation between MPI on day 2 and the peak systolic coronary flow velocity $(r=-0.54, \mathrm{p}<0.02)$. Moreover, MPI on day 2 was negatively correlated with the coronary diastolic deceleration time and positively correlated with the coronary diastolic deceleration rate (fig 3). At one week after PCI, MPI was also larger in patients who had early systolic retrograde coronary flow than in patients who did not have early systolic

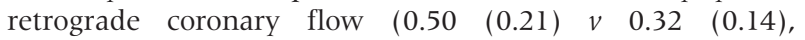
$\mathrm{p}<0.04)$.

\section{MPI and left ventricular function}

A significant positive correlation was observed between MPI on day 2 and the peak concentrations of creatine kinase and creatine kinase MB isoenzyme (table 2). LVEDP and LVEDV before PCI were not correlated with MPI on day 2. LVEF before PCI was only weakly correlated with MPI on day 2 $(r=-0.35, \mathrm{p}<0.05)$. A-WMSI just after PCI was significantly correlated with MPI on day $2(r=0.47, \mathrm{p}<0.008)$. Although MPIs measured immediately after PCI $(0.37(0.13))$ and one week after PCI $(0.42(0.30))$ were not correlated with

Table $3 \mathrm{MPI}$ on day 2 and left ventricular functional variables

\begin{tabular}{|c|c|c|c|}
\hline & \multicolumn{2}{|c|}{ MPI on day 2} & \multirow[b]{2}{*}{ p Value } \\
\hline & $\leqslant 0.35$ & $>0.35$ & \\
\hline \multicolumn{4}{|l|}{ A-WMSI } \\
\hline Just after $\mathrm{PCl}$ & $2.80(0.12)$ & $2.91(0.22)$ & 0.07 \\
\hline 1 Week after PCl & $2.52(0.58)$ & $2.97(0.20)$ & $<0.03$ \\
\hline 6 Months after $\mathrm{PCl}$ & $1.85(0.63)$ & $2.60(0.71)$ & $<0.02$ \\
\hline \multicolumn{4}{|l|}{ LVEDP $(\mathrm{mm} \mathrm{Hg})$} \\
\hline Just after $\mathrm{PCl}$ & $30(5)$ & $30(8)$ & 0.70 \\
\hline 1 Week after $\mathrm{PCl}$ & $20(5)$ & $24(6)$ & 0.09 \\
\hline 6 Months after PCl & $21(5)$ & $25(5)$ & 0.08 \\
\hline \multicolumn{4}{|l|}{ LVEF (\%) } \\
\hline Just after $\mathrm{PCl}$ & $46(7)$ & $42(10)$ & 0.26 \\
\hline 1 Week after PCl & $54(12)$ & 42 (12) & $<0.02$ \\
\hline 6 Months after $\mathrm{PCl}$ & $66(12)$ & 49 (19) & $<0.02$ \\
\hline \multicolumn{4}{|l|}{ LVEDV (ml) } \\
\hline Just after $\mathrm{PCl}$ & $123(26)$ & $129(16)$ & 0.47 \\
\hline 1 Week after $\mathrm{PCl}$ & $121(34)$ & $144(26)$ & 0.06 \\
\hline 6 Months after $\mathrm{PCl}$ & $114(36)$ & $144(43)$ & 0.09 \\
\hline
\end{tabular}

the percentage changes of A-WMSI, LVEDP, LVEF, and LVEDV at one week and six months after PCI, MPI measured on day $2(0.34(0.13))$ was significantly correlated with these parameters (table 2). Table 3 shows the actual changes in LVEDP, LVEF, and LVEDV in patients with MPI $\leqslant 0.35$ and $>0.35$.

\section{DISCUSSION}

This is the first study to show that the MPI on day 2 reflects myocardial perfusion after coronary revascularisation and that MPI can be used to predict the outcome of left ventricular systolic and diastolic function and the extent of time dependent left ventricular dilatation in the remote period after acute anterior MI. A recent study ${ }^{6-9}$ showed that MPI reflects the severity of left ventricular dysfunction and has prognostic value in patients with acute MI. Moller and colleagues $^{7}$ showed that the Doppler derived MPI measured on day $\mathrm{l}$ is a predictor of early left ventricular dilatation and cardiac death after a first acute MI. Most of the patients in these studies, however, did not undergo emergency direct PCI and it is uncertain whether the culprit coronary lesions were successfully recanalised. All of our study patients were successfully treated with PCI within six hours after the onset of symptoms and were alive during a one year follow up after MI. Furthermore, other studies included patients with various infarction sites and only $34-52 \%$ with acute anterior MI. Although Poulsen and colleagues $^{6}$ observed serial changes of MPI in acute MI, they measured MPI only on days $\mathrm{l}$ and 5 after admission and observed only a weak correlation between MPI and peak creatine kinase MB isoenzyme. Recent studies ${ }^{15} 16$ failed to find any independent prognostic impact of global MPI in patients with acute MI. Baseline LVEF, left ventricular systolic volume index, and $\mathrm{E}$ wave deceleration time emerged as strong independent predictors of death and heart failure. However, those studies also examined patients with various infarction sites and without successful direct PCI.

Mean MPIs in the prior studies were greater than the value measured in our study. In several previous studies the normal mean (SD) of MPI has been determined to be 0.35 (0.05). Indeed, the normal mean of MPI in patients with no history of heart diseases, normal blood pressure, and normal two dimensional and Doppler echocardiography in our institute was $0.34(0.05)$. It is unknown exactly why MPI in many patients with anterior MI was lower than what is seen in 
healthy people. One possibility is that left ventricular functions are overcompensated by non-infarcted and salvaged myocardium during the 24 hours after successful PCI. In support of this view, MPI was significantly less one day after PCI than one week after PCI.

Previous studies with a Doppler guidewire ${ }^{14}$ 17-19 have shown that a low average systolic peak velocity, a rapid diastolic flow velocity deceleration time, a high diastolic flow velocity deceleration rate, and the existence of early systolic retrograde coronary flow immediately after PCI reflect more severe microvascular damage in the area at risk and are related to no reflow phenomenon. The coronary blood flow pattern was reported to be only slightly improved during the 24 hours after PCI. ${ }^{17}$ Previous studies of patients with acute $\mathrm{MI}^{2-5}$ have suggested that the optimum time to estimate microvascular integrity by myocardial contrast echocardiography for predicting myocardial viability may be one day after revascularisation. Our MPI results support these reports of left ventricular systolic function after PCI. We showed that the extent of time dependent left ventricular dilatation may also be determined by one day after recanalisation.

Postinfarction left ventricular remodelling is influenced by various mechanical, neurohormonal, and genetic factors. This process has been arbitrarily divided into an early phase during which the infarct zone expands and a late phase associated with time dependent left ventricular dilatation, distortion of ventricular shape, and mural hypertrophy. Although late remodelling is influenced by various factors including the renin-angiotensin system (providing the rationale for treatment with angiotensin converting enzyme inhibitors), left ventricular time dependent dilatation and deterioration of left ventricular contractility in the remote period may be determined in the early stage after successful revascularisation. Previous studies ${ }^{2}{ }^{3}$ have suggested that microvascular injury was already partially healed and that neovascularisation within the infarcted area had begun by day 2. Indeed, our Doppler guidewire study showed that MPI on day 2 reflected the damage to the coronary microvasculature immediately after PCI. Therefore, there seems to be a need for more effective methods of preventing microvascular injury within 24 hours after coronary revascularisation. Nevertheless, left ventricular diastolic dysfunction after MI could not be completely determined in the early phase because Doppler derived MPI on day 2 did not predict the change in LVEDP during six months in our study.

MPI is a simply measurable Doppler derived index of combined systolic and diastolic myocardial performance, ${ }^{6-9} 2021$ which is easily applicable to the assessment of overall right and left ventricular myocardial function. In brief, left ventricular systolic dysfunction lengthens the pre-ejection period and shortens the ejection time, so that the pre-ejection period to ejection time ratio increases. The value of the time constant of left ventricular relaxation increases through all stage of diastolic dysfunction, so that isovolumic relaxation time becomes prolonged with abnormal relaxation. Shortened ejection time with premature mitral valve closure and prolongation of isovolumic contraction time compensate for increased left ventricular filling pressure. As a result, MPI increases and provides functional information. ${ }^{22}$ The advantages of this index are reported to be less dependence on the heart rate and blood pressure, no influence of geometric changes of the ventricle, easy quantitative assessment, suitability for follow up studies, and cost effectiveness. Most patients with acute MI can be easily evaluated with this index when compared with other methods such as myocardial contrast echocardiography, dobutamine stress echocardiography, Doppler guidewire studies, and thallium201 scintigraphy. ${ }^{2} 1417$ 23-25 However, the MPI is unsuitable for the evaluation of left ventricular function in patients with atrial fibrillation, atrial flutter, atrioventricular block, severe mitral or aortic valvar disease, and severe pericardial effusion because these factors significantly influence the patterns of left ventricular inflow and outflow.

\section{Study limitations}

We excluded patients with atrial fibrillation, atrial flutter, atrioventricular block, severe mitral or aortic valvar disease, and severe pericardial effusion. As with other methods, the MPI may be affected to some degree by loading conditions. ${ }^{26}$ This is the limitation of our study of Doppler derived MPI.

\section{Conclusions}

Doppler derived MPI on day 2 after early successful coronary revascularisation may be representative of the early coronary microvascular state and can predict the eventual improvement of left ventricular systolic function and late remodelling in patients with their first anterior acute MI. Further investigations seem to be needed to develop more effective methods for preventing microvascular injury within 24 hours after coronary revascularisation.

\section{Authors' affiliations}

M Kato, K Dote, S Sasaki, K Goto, H Takemoto, S Habara,

D Hasegawa, Department of Cardiology, Hiroshima City Asa Hospital, Hiroshima, Japan

\section{REFERENCES}

1 Sutton MG, Sharpe N. Left ventricular remodeling after myocardial infarction: pathophysiology and therapy. Circulation 2000;101:2981-8.

2 Sakuma T, Hayashi Y, Sumii K, et al. Prediction of short- and intermediateterm prognoses of patients with acute myocardial infarction using myocardial contrast echocardiography one day after recanalization. J Am Coll Cardiol 1998:32:890-7.

3 Sakuma T, Otsuka M, Okimoto T, et al. Optimal time for predicting myocardial viability after successful primary angioplasty in acute myocardial infarction: a study using myocardial contrast echocardiography. Am J Cardiol 2001;87:687-92.

4 Lepper W, Sieswerda GT, Vanoverschelde JL, et al. Predictive value of markers of myocardial reperfusion in acute myocardial infarction for follow-up of left ventricular function. Am J Cardiol 2001;88:1358-63.

5 Lepper W, Hoffmann R, Kamp O, et al. Assessment of myocardial reperfusion by intravenous myocardial contrast echocardiography and coronary flow reserve after primary percutaneous transluminal coronary angioplasty in patients with acute myocardial infarction. Circulation 2000;101:2368-74.

6 Poulsen SH, Jensen SE, Nielsen JC, et al. Serial changes and prognostic implications of a Doppler-derived index of combined left ventricular systolic and diastolic myocardial performance in acute myocardial infarction. Am J Cardiol 2000;85: 19-25.

7 Moller JE, Sondergaard E, Poulsen SH, et al. The Doppler echocardiographic myocardial performance index predicts left-ventricular dilation and cardiac death after myocardial infarction. Cardiology 2001;95:105-11.

8 Szymanski P, Rezler J, Stec S, et al. Long-term prognostic value of an index of myocardial performance in patients with myocardial infarction. Clin Cardiol 2002;25:378-83.

9 Moller JE, Egstrup K, Kober L, et al. Prognostic importance of systolic and diastolic function after acute myocardial infarction. Am Heart $J$ 2003; 145: 147-53.

10 Schiller NB, Shah PM, Crawford M, et al. Recommendations for quantitation of the left ventricle by two-dimensional echocardiography: American Society of Echocardiography Committee on Standards, Subcommittee on Quantitation of Two-Dimensional Echocardiograms. J Am Soc Echocardiogr 1989;2:358-67.

11 Smart SC, Sawada S, Ryan T, et al. Low-dose dobutamine echocardiography detects reversible dysfunction after thrombolytic therapy of acute myocardial infarction. Circulation 1993;88:405-15.

12 Kennedy JW, Trenholme SE, Kasser IS. Left ventricular volume and mass from single-plane cineangiogram: a comparison of anteroposterior and right anterior oblique methods. Am Heart J 1970;80:343-52.

13 Haase J, Di Mario C, Slager CJ, et al. In-vivo validation of on-line and off-line geometric coronary measurements using insertion of stenosis phantoms in porcine coronary arteries. Cathet Cardiovasc Diagn 1992;27:16-27.

14 Iwakura K, Ito H, Takiuchi S, et al. Alternation in the coronary blood flow velocity pattern in patients with no reflow and reperfused acute myocardial infarction. Circulation 1996;94:1269-75.

15 Schwammenthal E, Adler Y, Amichai K, et al. Prognostic value of global myocardial performance indices in acute myocardial infarction: comparison to measures of systolic and diastolic left ventricular function. Chest 2003; 124:1645-51. 
16 Hole T, Skaerpe T. Myocardial performance index (Tei index) does not reflect long-term changes in left ventricular function after acute myocardial infarction. Echocardiography 2003;20:1-7.

17 Lepper W, Sieswerda GT, Franke A, et al. Repeated assessment of coronary flow velocity pattern in patients with first acute myocardial infarction. J Am Coll Cardiol 2002;39:1283-9.

18 Kawamoto T, Yoshida K, Akasaka T, et al. Can coronary blood flow velocity pattern after primary percutaneous transluminal coronary angiography predict recovery of regional left ventricular function in patients with acute myocardial infarction? Circulation 1999;100:339-45.

19 Wakatsuki T, Nakamura M, Tsunoda T, et al. Coronary flow velocity immediately after primary coronary stenting as a predictor of ventricular wall motion recovery in acute myocardial infarction. J Am Coll Cardiol 2000;35:1835-41.

20 Tei C, Nishimura RA, Seward JB, et al. Noninvasive Doppler-derived myocardial performance index: correlation with simultaneous measurements of cardiac catheterization measurements. J Am Soc Echocardiogr 1997; 10:169-78
21 Yeo TC, Dujardin KS, Tei C, et al. Value of a Doppler-derived index combining systolic and diastolic time intervals in predicting outcome in primary pulmonary hypertension. Am J Cardiol 1998;81:1157-61.

22 Szymanski P, Rezler J, Stec S, et al. Long-term prognostic value of an index of myocardial performance in patients with myocardial infarction. Clin Cardiol 2002;25:378-83.

23 Botvinick EH, Perez-Gonzalez JF, Dunn R, et al. Late prognostic value of scintigraphic parameters of acute myocardial infarction size in complicated myocardial infarction without heart failure. Am J Cardiol 1983:51:1045-51.

24 Picano E, Sicari R, Landi $\mathrm{P}$, et al. Prognostic value of myocardial viability in medically treated patients with global left ventricular dysfunction early after an acute uncomplicated myocardial infarction: a dobutamine stress echocardiographic study. Circulation 1998;98:1078-84.

25 Sicari R, Varga A, Picano $E$, et al. Comparison of combination of dipyridamole and dobutamine during echocardiography with thallium scintigraphy to improve viability detection. Am J Cardiol 1999;83:6-10.

26 Moller JE, Poulsen SH, Egstrup K. Effects of preload alterations on a new Doppler echocardiographic index of combined systolic and diastolic performance. J Am Soc Echocardiogr 1999;135:1065-72.

\section{IMAGES IN CARDIOLOGY}

\section{The "tricolore" sign of heart failure}

A 73 year old male patient with ischaemic cardiomyopathy and congestive heart failure (New York Heart Association functional class IV) presented with cachexia and chronic pleural effusion. He was sent for abdominal and thoracic computed tomography (CT) scans to rule out malignancy. Aside from cardiomegaly and pleural effusion the CT showed few developmental cysts of the liver but no suspicion of a malignant tumour.

The images were unusual for a distinctive trilaminar contrast density in the inferior vena cava (panel A) that was found 70 seconds after dye injection into the right cubital vein. The opaque bottom layer was explained by a substantial retrograde flow of almost undiluted contrast medium below the level of the right atrium. The middle layer consisted of contrasted blood returning from both renal veins into the

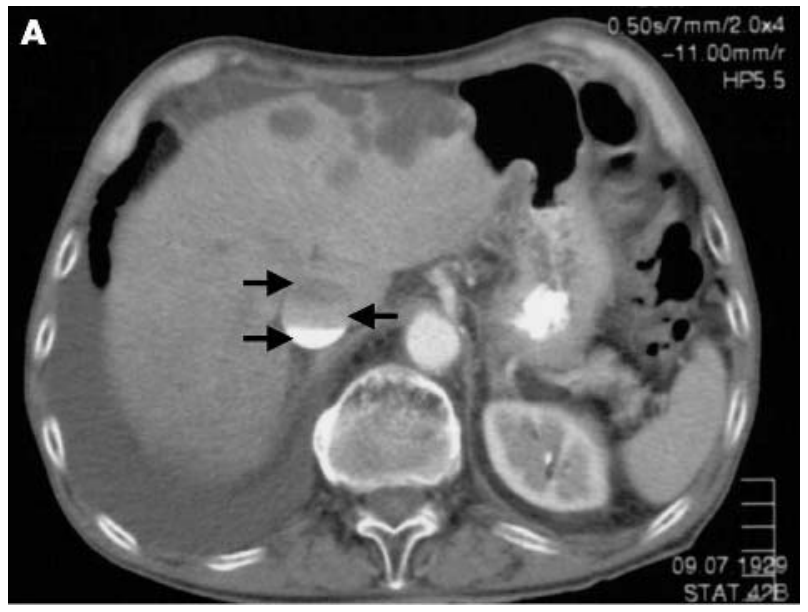

Abdominal CT scan $70 \mathrm{~s}$ after antecubital intravenous administration of $70 \mathrm{ml}$ contrast medium (flow $2 \mathrm{ml} / \mathrm{s}$ ) in a patient with low output heart failure. Note the inferior vena cava displays three distinct layers of contrasted blood (61 HU, $126 \mathrm{HU}$, and $785 \mathrm{HU}$, arrowheads), indicating slow laminar blood flow. inferior vena cava after first passing through the kidneys. The top layer represented poorly contrasted blood mainly returning from the lower extremities.

This study is remarkable because there was almost no admixture between these three distinctly contrasted layers. We attribute this "tricolore phenomenon" of heart failure to the physicochemical properties of the contrast medium in coherence with slow laminar flow secondary to low cardiac output.

\section{H Liptau \\ J Gellissen H Schunkert heribert.schunkerł@innere2.uni-luebeck.de}

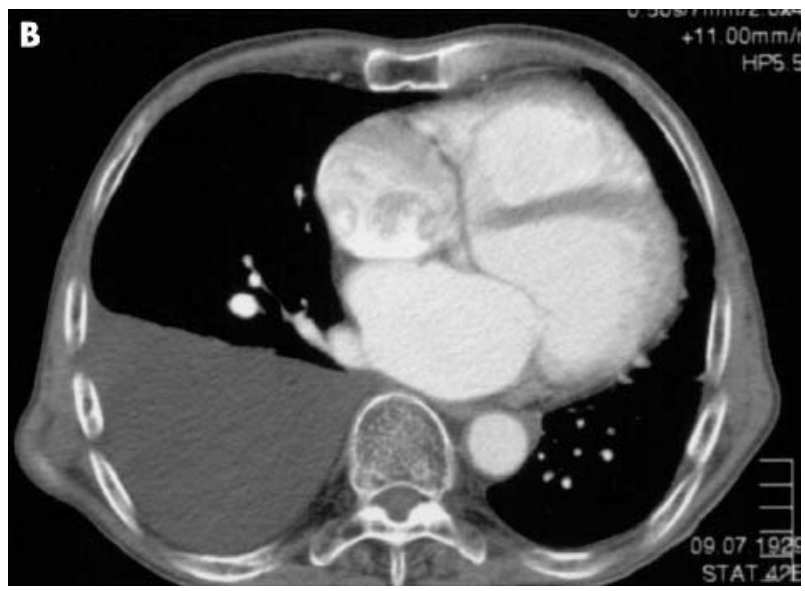

Thoracic CT scan depicting basal parts of the enlarged heart. A seemingly turbulent influx of contrast medium is seen in the right atrium while the other cardiac cavities display a homogeneous density. 